\title{
Activité en strontium go d'une eau potable de la région parisienne, de 1963 à 1972
}

\author{
L. JEANMAIRE, F. PATTI, R. GROS \\ (Manuscrit reçu le 28 novembre 1975)
}

\begin{abstract}
RÉSUMÉ
Le strontium 90 a été dosé, de 1963 à 1972, dans une eau potable du sud de la région parisienne. L'activité, généralement inférieure au picocurie par litre, est liée au débit de la Seine, et décroît avec une période d'environ cinq ans.
\end{abstract}

\begin{abstract}
Strontium 90 was determined in tap water of the Southern Paris area from 1963 to 1972 . Activities usually below $1 \mathrm{pCi} / \mathrm{l}$ were related to the Seine river flow and decreased with a half-life of about five years.
\end{abstract}

Dans le cadre du contrôle de la pollution radioactive du milieu, le strontium 90 est dosé mensuellement dans une eau potable depuis le mois d'avril 1963.

Cette eau prélevée dans un laboratoire du Centre d'Études nucléaires de Fontenay-aux-Roses provient de l'usine de Choisy-le-Roi qui traite de l'eau de Seine pour la rendre propre à la consommation, et alimente en eau potable le sud de la région parisienne.

Un échantillon de 41 d'eau du robinet est prélevé chaque jour et passé sur une colonne de résine cationique. $\mathrm{A}$ la fin du mois, la résine est régénérée, et le strontium dosé dans l'éluat [1].

Les résultats en picocuries de strontium 90 par litre d'eau sont indiqués dans le tableau I et la figure 1 . On constate, au cours du temps, une décroissance générale de l'activité, avec quelques pics nettement marqués. Bien que

$\left(^{*}\right)$ Commissariat à l'Énergie atomique, Département de Protection, Service de Protection Sanitaire, B. P. $n^{\circ}$ 6, 92260 Fontenay-aux-Roses.

RADIOPROTECTION, VOL. $11-\mathrm{N}^{\circ} 1$ 
les mesures présentées ne remontent pas au delà de 1963, il est probable que les premières valeurs indiquées correspondent au maximum de la concentration de ${ }^{90} \mathrm{Sr}$ dans cette eau, car c'est en 1963 que les retombées radioactives ont été les plus fortes en France; des mesures analogues sur l'eau de la ville de New York ont présenté un maximum de 2,14 pCi/l au mois de juillet 1963 [2].

TABLEAU I

TENeURS EN STRONTIUM 90 D'UNe EAU POTABLE DE LA RÉGION PARISIENNE OBTENUE A PARTIR D'EAU DE SEINE D'AVRIL 1963 A DÉCEMBRE 1972

\begin{tabular}{|l|c|c|c|c|c|c|c|c|c|c|}
\hline \multicolumn{1}{|c|}{$\begin{array}{c}\text { Echantillons } \\
\text { mensuels }\end{array}$} & \multicolumn{10}{|c|}{ Activités en pCi/1 } \\
\hline & 1963 & 1964 & 1965 & 1966 & 1967 & 1968 & 1969 & 1970 & 1971 & 1972 \\
\cline { 2 - 11 } & - & 0,50 & 0,70 & 0,95 & 0,45 & 1,10 & 0,40 & 0,30 & 0,21 & 0,18 \\
JANVIER & - & 0,80 & 0,70 & 0,95 & 0,42 & 0,78 & 0,37 & 0,60 & 0,35 & 0,29 \\
FEVRIER & - & 0,83 & 0,70 & - & 0,38 & 0,33 & 0,42 & 0,54 & 0,25 & 0,22 \\
MARS & 0,98 & 1,00 & 0,90 & - & 0,25 & 0,24 & 0,25 & 0,38 & 0,22 & 0,18 \\
AVRIL & - & 0,86 & 0,60 & - & 0,23 & 0,38 & 0,35 & 0,24 & 0,27 & 0,23 \\
MAI & 2,00 & 0,93 & 0,81 & - & 0,23 & 0,30 & 0,33 & 0,23 & 0,35 & 0,20 \\
JUIN & - & 0,75 & 0,43 & - & 0,24 & 0,44 & 0,32 & 0,24 & 0,31 & 0,16 \\
JUILLET & 1,96 & 0,64 & 0,46 & 0,55 & 0,34 & 0,31 & - & - & 0,28 & - \\
AOUT & 1,20 & 0,68 & 0,58 & 0,37 & 0,40 & 0,42 & 0,25 & 0,22 & 0,23 & 0,20 \\
SEPTEMBRE & 0,83 & 0,75 & 0,62 & 0,30 & 0,37 & 0,41 & 0,23 & 0,26 & - & 0,15 \\
OCTOBRE & 1,12 & 0,66 & 0,43 & 0,42 & 0,46 & 0,37 & 0,20 & 0,23 & 0,26 & 0,34 \\
NOVEMBRE & 1,23 & 0,60 & 1,20 & 0,83 & 0,46 & 0,30 & 0,30 & 0,30 & - & 0,27 \\
DECEMBRE & 1,33 & 0,75 & 0,68 & 0,62 & 0,34 & 0,45 & 0,31 & 0,30 & 0,27 & 0,22 \\
\hline MOYENNE & \multicolumn{10}{|c|}{} \\
\hline
\end{tabular}

On peut se demander si les pics d'activité suivants sont dus également à de nouvelles explosions nucléaires. Les tirs aériens dans l'hémisphère Nord ont cessé fin 1962, à l'exception de 13 tirs chinois [3] qui ne représentent qu'un faible pourcentage des tirs aériens précédents effectués par d'autres pays; il n'est pas du tout évident que l'on puisse relier les maximums observés sur la courbe à des explosions aériennes effectuées durant la même période, et il semble bien que l'année 1963 doive être considérée à part. La période apparente de décroissance calculée entre 1964 et 1972 est d'environ 4,5 années.

Les explosions aériennes effectuées depuis 1964, n'ayant, apparemment, que peu d'influence sur les variations de la contamination de l'eau de Seine, nous nous sommes demandé si l'importance du débit de ce fleuve jouait un rôle; nous avons remarqué que trois périodes de débit maximal du fleuve en 1966, 1968 et 1970, correspondent à des pics d'activité dans l'eau (tableau II). Le coefficient de corrélation linéaire entre débit et activité est faible $(0,26)$, si l'on prend toutes les données de 1963 à 1972, et s'améliore notablement $(0,49)$, lorsqu'on supprime l'année 1963. Le maximum de corrélation est recher- 


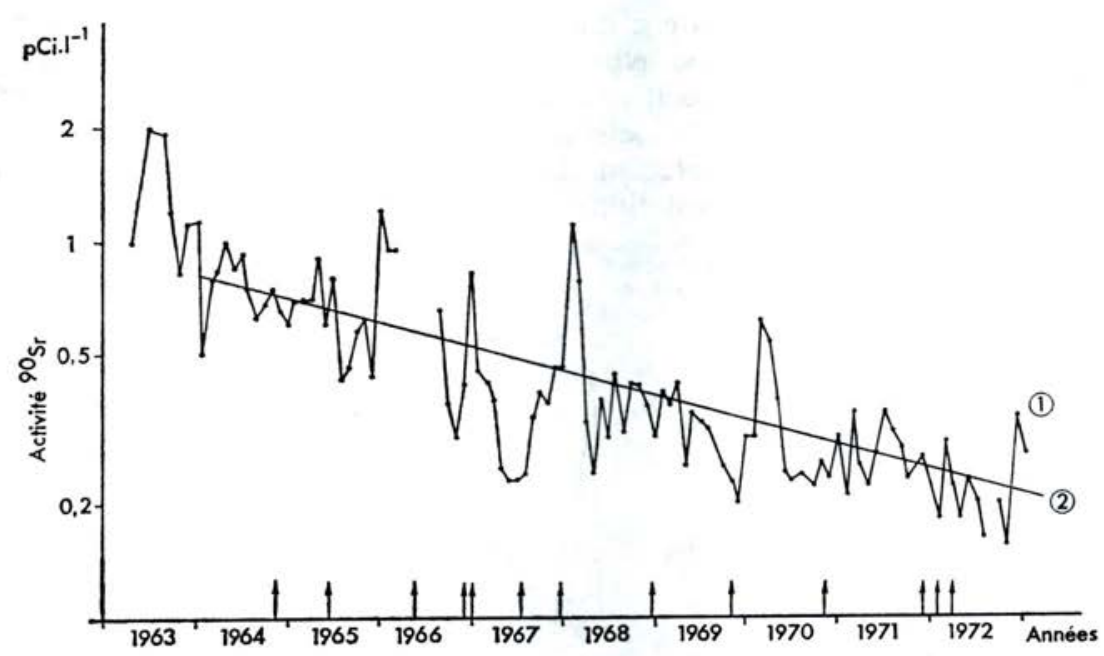

FIG. 1. - La courbe 1 représente la variation des concentrations en ${ }^{90} \mathrm{Sr}$ par litre d'eau, d'avril 1963 à décembre 1972. La courbe 2 est la droite des moindres carrés indiquant la décroissance de janvier 1964 à décembre 1972. Les flèches correspondent aux explosions aériennes effectuées dans l'hémisphère Nord.

ché, en affectant les valeurs d'activité de divers coefficients de décroissance; le coefficient maximal de corrélation $(0,73)$ est obtenu en attribuant à l'activité une période de décroissance de cinq ans ( fig. 2). La liaison positive entre l'activité et le débit est donc bien évidente, quoique surprenante au premier

\section{TABLEAU II}

Débits mensuels de La Seine a Choisy-Le-Roi en mètres cubes par seconde

\begin{tabular}{|l|r|r|r|r|r|r|r|r|r|r|r|}
\hline \multicolumn{1}{|c|}{ Mois } & 1963 & 1964 & 1965 & 1966 & 1967 & 1968 & 1969 & 1970 & 1971 & 1972 & 1973 \\
\hline JANVIER & 110 & 73 & 166 & 671 & 425 & 829 & 316 & 166 & 125 & 71 & 86 \\
FEVRIER & 68 & 105 & 198 & 651 & 267 & 498 & 269 & 750 & 196 & 144 & 288 \\
MARS & 160 & 183 & 211 & 360 & 279 & 307 & 373 & 721 & 146 & 104 & 160 \\
AVRIL & 192 & 225 & 304 & 316 & 144 & 185 & 193 & 555 & 111 & 92 & 75 \\
MAI & 113 & 136 & 226 & 234 & 97 & 273 & 280 & 290 & 102 & 73 & 95 \\
JUIN & 135 & 51 & 189 & 103 & 65 & 147 & 157 & 123 & 111,5 & 32,9 & 73 \\
JUILLET & 94 & 25 & 82 & 112 & 60 & 108 & 96 & 123 & 65,5 & 37,2 & 30,7 \\
AOUT & 66 & 19,5 & 57 & 115 & 69 & 109 & 88 & 107 & 70 & 80 & 32,9 \\
SEPTEMBRE & 67 & 24 & 112 & 80 & 77 & 211 & 83 & 97 & 61 & 62 & 39,9 \\
OCTOBRE & 59 & 39 & 142 & 82 & 93 & 210 & 67 & 97 & 40,3 & 41,2 & 35,5 \\
NOVEMBRE & 141 & 36 & 149 & 103 & 169 & 186 & 75 & 84 & 54 & 175 & 41,5 \\
DECEMBRE & 117 & 56 & 592 & 412 & 200 & 316 & 131 & 115 & 55 & 183 & 202 \\
\hline
\end{tabular}

voL. $11-\mathrm{N}^{\circ} 1$ 
abord, car on pouvait s'attendre à une relation inverse entre ces deux paramètres. Nous proposons comme hypothèse non vérifiée l'explication partielle suivante : les maximums de débit sont consécutifs à des pluies importantes; dans ce cas, l'eau de pluie qui, elle-même, contient une certaine activité, n'a pas le temps de filtrer normalement dans le sol et, au cours du ruissellement, elle peut encore remettre en solution une partie du strontium radioactif du dépôt superficiel.

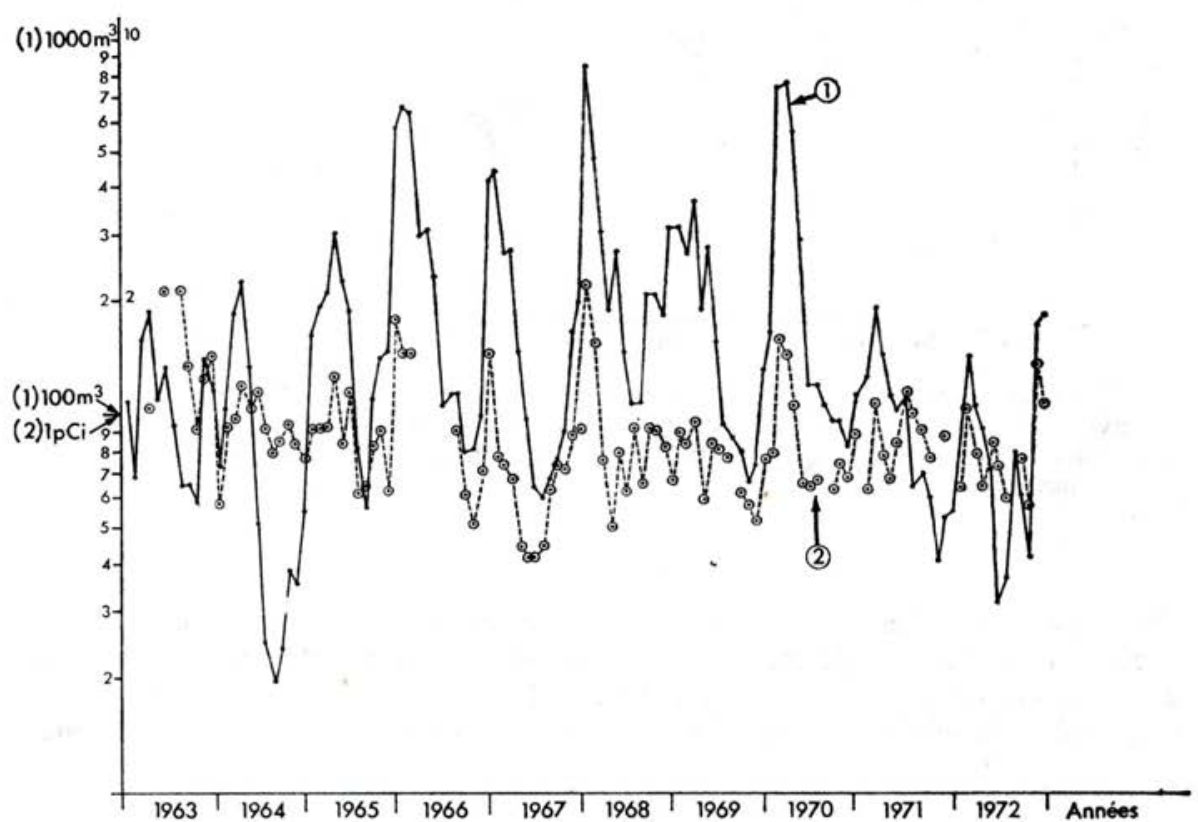

FIG. 2

(1) Courbe du débit moyen mensuel de la Seine en $\mathrm{m}^{3} \mathrm{~s}^{-1}$.

(2) Courbe de l'activité en ${ }^{90} \mathrm{Sr}$ par litre d'eau corrigée de la décroissance (période admise : cinq ans).

Le tableau III permet de comparer l'activité en ${ }^{90} \mathrm{Sr}$ de l'eau à celle du lait, aliment peu contaminé qui est, cependant, la source principale de la contamination en ${ }^{90} \mathrm{Sr}$ chez l'homme [4]. L'activité de l'eau a rarement dépassé le niveau du picocurie par litre, et elle est largement inférieure d'un ordre de grandeur à celle du lait. Dans le même tableau, les valeurs de ${ }^{90} \mathrm{Sr}$ par gramme de calcium ont été calculées en admettant une concentration uniforme de $80 \mathrm{mg}$ de calcium par litre d'eau; les valeurs pour l'eau sont encore environ deux fois plus faibles que celles du lait consommé dans la région parisienne. En admettant une consommation journalière d'un litre d'eau, l'apport à l'organisme en ${ }^{90} \mathrm{Sr}$ par l'eau du robinet représenterait environ 10 p. cent de celui dû au lait. 
TABLEAU III

TENEURS EN STRONTIUM 90 D'UNE EAU POTABLE ET DE LAIT CONSOMMÉ DANS LA RÉGION PARISIENNE

\begin{tabular}{|c|c|c|c|}
\hline Années & $\begin{array}{c}\text { Eau : }{ }^{90} \mathrm{Sr} \\
\mathrm{pCi} / 1\end{array}$ & $\begin{array}{c}\mathrm{Eau}:{ }^{90}{ }_{\mathrm{Sr}} \\
\mathrm{pCi} / \mathrm{g} \text { de Ca }\end{array}$ & $\begin{array}{c}\text { Lait }[4] \cdot{ }^{90} \mathrm{Sr} \\
\mathrm{pCi} / \mathrm{g} \text { de Ca }\end{array}$ \\
\hline 1963 & 1,33 & 16,5 & 41 \\
1964 & 0,75 & 9,4 & 28 \\
1965 & 0,68 & 8,5 & 21,4 \\
1966 & 0,62 & 7,7 & 12,6 \\
1967 & 0,34 & 4,3 & 10,9 \\
1968 & 0,45 & 5,6 & 8,8 \\
1969 & 0,31 & 3,9 & 7,8 \\
1970 & 0,30 & 3,8 & 6 \\
1971 & 0,21 & 2,5 & 5,3 \\
\hline 1972 & 0,18 & 2,2 & \\
\hline
\end{tabular}

Note. -1 litre de lait contient environ 1,1 gramme de calcium.

\section{REMERCIEMENTS}

Nous remercions $M^{\text {lles }}$ Bullier et LAPORTE de leur collaboration technique, ainsi que la Direction de la Production de la Compagnie générale des Eaux qui nous a transmis les informations sur le débit de la Seine à Choisy-le-Roi.

\section{BIBLIOGRAPHIE}

[1] Jeanmaire L., Patti F., Bullier D. Dosage radiochimique du ${ }^{90} \mathrm{Sr}$ sur des volumes importants d'eau potable. Rapport CEA-R-2844, 1965.

[2] États-Unis, Health and Safety Laboratory report HASL-278, 1974, C 5.

[3] ZANDER I., ARASKog R. Nuclear explosions 1945-1972, basic data. FOA-4505-A1, 1973.

[4] Coulon R., Madelmont C. Contamination des os humains et du régime alimentaire par le strontium 90. Essai d'établissement d'un modèle de transfert. Dans Contamination par radionucléides ostéotropes et radioprotection, Grenoble, 1-5 février 1971. Montrouge, Société française de Radioprotection, 1971, 218-251.

VOL. $11-\mathrm{N}^{\circ} 1$ 